\title{
EVALUACIÓN DEL DOCENTE UNIVERSITARIO. UNA VISIÓN INSTITUCIONAL
}

Héctor Eli Rizo Moreno

Universidad Autónoma de Occidente, Colombia

\section{CONSIDERACIONES EN TORNO AL HECHO EVALUATIVO}

La educación juega el doble papel de conservar los valores y principios esenciales de la sociedad y al tiempo el de generar las condiciones desde las cuales puedan germinar los cambios que le permitan a la sociedad evolucionar hacia nuevos desarrollos y formas de vida. En este doble papel, que en ocasiones se armonizan y en otras existen en forma antagónica, se presentan las tensiones de los sistemas educativos y se enmarca el funcionamiento de las instituciones educativas.

Así pues, las instituciones educativas tradicionalmente se han movido desde dos marcos bien definidos: por un lado, desde el reconocimiento de la sociedad merced al papel que juegan en torno al progreso de la humanidad y por el otro desde las permanentes influencias, presiones y exigencias originadas en diferentes esferas de poder.

En uno de ellos pernocta el reconocimiento que les hace la humanidad como espacios de saber en el cual coexisten intelectuales, detentadores de saberes y por ende espacios válidos en los cuales es deseable y necesario que ingresen aquellos que requieren ser formados. En palabras del profesor Páramo ${ }^{1}$ "Las personas y los pueblos perecen o son esclavos de la naturaleza o de otros hombres cuando no son dueños intelectuales de sí mismos, porque solamente, siendo dueños de sí mismos pueden saber quienes son, cuál es su historia, cuál es su universo, cuáles son sus recursos y cuál es su necesidad; e igualmente, comprender cuáles son sus limitaciones y peligros, y cuales son los rumbos que no deben seguir y las empresas que por necias, fútiles o proporcionadas no deben acometer. De allí que, para todas las culturas, los sabios y algo como el Calmécac o la Academia es una necesidad" y continúa diciendo "Las Universidades fueron fundadas por nuestra cultura para satisfacer su necesidad de sabios entendidos como aquellos que pueden ver más allá de la apariencia, porque la generalidad de los seres humanos depende de la apariencia para reconocer a los tipos humanos de su propia sociedad'. ${ }^{2}$

En el otro marco aparecen las clases dirigentes a quienes les interesa, por obvias razones, el papel de perpetuador del statu quo incluyendo en ello exigencias a las instituciones para que preparen a las personas con los conocimientos, habilidades y destrezas que más convenga a sus intereses.

Uno de los diferenciadores entre los diferentes países, bien podría ser el marco que se privilegia. Los países como los nuestros no plasman la importancia que dicen asignarle a la educación en políticas y

\footnotetext{
${ }^{1}$ Vicerrector Académico de la Universidad Nacional de Colombia

${ }^{2}$ Páramo, G. Sentido cultural de la autonomía universitaria y de la vigilancia de su calidad, Editado por Consejo Nacional de Acreditación, Colombia, Julio 2002.
} 
estrategias que permitan su fortalecimiento, en parte porque sus propios gobiernos no gozan de la autonomía suficiente para ejercer su derecho a la libre autodeterminación.

En esta sociedad globalizada en la cual los estados catalogados del tercer mundo, carecen de independencia y de libertad de acción en todos los aspectos, los peligros de disolución ${ }^{3}$ han dejado de ser una voz de alarma de algunos sectores minoritarios y progresistas para convertirse en una realidad patente con desarrollos cada vez más dolorosos.

Políticamente; con la llamada desaparición de la guerra fría y el descarnado, violento, e inhumano pero predecible resurgimiento de la guerra terrorista, el énfasis policivo de tipo represivo ha polarizado el mundo, orientándolo hacia la hegemonía de un solo país. Actualmente la preocupación no se centra en el desarrollo social de la humanidad o la lucha contra las inequidades y la pobreza en la que se sume la gran mayoría de la población sino en la defensa mundial contra el terrorismo.

A su nombre, se asiste con pasmosa pasividad a las masacres que comete un estado fuerte militarmente, además apoyado por la principal potencia mundial, contra una indefensa población; se prepara todo el mundo "civilizado" a apoyar otras acciones contra pueblos indefensos, todo en nombre de la cruzada mundial contra el terrorismo. Los pueblos que hemos sufrido y seguimos sufriendo los embates de tipo terrorista, somos conscientes de su irracionalidad y de lo importante que es su eliminación, pero moralmente deberíamos sentirnos impedidos para apoyar ese terrorismo de estado, reflejado en matanzas indiscriminadas con el cual se pretende combatirlo.

En este contexto la "diplomacia" mundial ya no se alarma ante los pedidos norteamericanos para que se plieguen acríticamente a sus designios ${ }^{4}$. Obviamente, país que no acceda será sometido a todo tipo de represalias. Europa, considerada una reserva moral de la humanidad, muy rara vez -tímida y pusilánimemente- deja escuchar su voz de inconformidad. Estamos pues desde el punto de vista político ante Estados que han perdido su libre autodeterminación.

Económicamente, las manifestaciones en el sentido de que la Banca Mundial imponía las políticas macroeconómicas de los países, siempre traducidas en la ampliación de la brecha entre ricos y pobres, han dejado de ser simples advertencias de algunos "marxistas" ${ }^{5}$. Hoy en día hablan por sí solos los hechos, los casos de Argentina y siempre los demás países latinoamericanos ponen en evidencia cómo actúa la estructura económica mundial y cómo se derrumba de fácil un país cuando los emporios financieros del mundo así lo determinan.

Culturalmente la globalización aceleradamente impone la perdida de los valores autóctonos para incorporar especialmente en la juventud diferentes valores culturales y modos de vida que en nada permiten reconstruir el tejido social que se debilita y constituye, tal vez, la última esperanza de cohesión nacional.

Vemos, entonces, que los parámetros externos determinan la calidad de vida y los desarrollos internos de los países y afectan profundamente sus propias estructuras. En la entrega inmisericorde de las

\footnotetext{
${ }^{3}$ En Colombia, el actual gobierno justifica una declaratoria de conmoción interior, esgrimiendo como principal argumento el peligro de disolución social en que se encuentra el país. Asume que con las herramientas represivas que, de esa declaratoria se derivan, puede combatir eficientemente la guerrilla, considerada por él, como su principal causa.

${ }^{4}$ Sin olvidarse que ellos impulsaron e impusieron los tribunales internacionales para juzgar delitos de lesa humanidad, exigieron con toda tranquilidad, a los países que apoyaran una resolución mediante la cual esos tribunales no puedan juzgar a los norteamericanos.

${ }^{5}$ Hasta ese nombre, otrora sinónimo de reflexión crítica y preocupación por el bienestar de los más desfavorecidos, tiene ahora, connotación peyorativa
} 
decisiones nacionales a la mirada inquisidora de otros organismos internacionales podemos asignar gran parte de la culpa de la disolución de la que hablábamos al principio de este apartado.

Así, por ejemplo, muy especialmente desde los años 70, ha sido inclemente la presión ejercida sobre nuestros países por parte de los diferentes organismos financieros para que se establezcan políticas de "racionalización del gasto". Políticas que, lejos de afectar al sector burocrático o militar, inexorablemente enfatizan en los sectores sociales y especialmente en el de la salud y en el de la educación, bajo dos formas específicas: Buscar la auto-sostenibilidad de las instituciones hospitalarias y controlar los productos educativos desde criterios de rentabilidad y eficiencia.

En este contexto se impulsa desde las esferas gubernamentales esquemas de rendición de cuentas, se fomentan los ejercicios de autoevaluación con perspectivas de acreditación y se invita a incorporar en las estructuras curriculares asignaturas y énfasis que contribuyan a generar una cultura de optimización de recursos de cualquier tipo. No es extraño entonces, que las actuales propuestas evaluativas abarquen decididamente otros ámbitos del mundo educativo y de manera específica las evaluaciones de desempeño, superando con creces el tradicional y casi exclusivo enfoque que se ha hecho sobre el aprendizaje.

Tal vez, la base desde la cual la evaluación encuentra su coherencia está constituida por la pregunta fundamental que le corresponde asumir: ¿Cómo cumple la educación la doble función de Preservar y conservar los valores fundamentales de la sociedad, y a su vez, generar las condiciones necesarias para posibilitar su propio desarrollo en una permanente búsqueda del mejoramiento de las posibilidades de existencia para todos sus miembros?

Como se ha visto, la pregunta no es posible abordarla sin tener presente el contexto social existente. Por ejemplo, en plena revolución industrial se requería que las instituciones educativas proporcionaran a los ciudadanos la formación que enfatizara en: a) La mecanización de rutinas, pues la mano de obra que se necesitaba debería ser competente en repetición acertada de actividades; b) La memorización, pues era necesario aprender y seguir instrucciones contenidas en manuales; c) La disciplina y obediencia, pues la industria necesitaba personas que supieran acatar y someterse a las ordenes de sus superiores.

Las estructuras educativas y los énfasis evaluativos se pueden analizar desde esta misma perspectiva. La división del trabajo y el énfasis punitivo de la evaluación pueden entenderse desde los trabajos de Taylor y de Henry Fayol publicados en "Administration Industrielle et Genérale" en 1916 que sintetizaban los procesos administrativos en planear, organizar, dirigir, coordinar y controlar; bajo su influencia aparecen también los términos: tecnología de la educación, diseño curricular, objetivos de aprendizaje o evaluación educativa, los cuales habrían de establecer un claro enfoque cuantitativo y una demarcación tecnológica de la evaluación.

En los años treinta, cuando la gran depresión originó un estancamiento generalizado en la educación y la necesidad de optimizar los recursos dedicados al desarrollo de los programas educativos, Ralph Tyler publica en 1942 su obra General Stament of Education y en 1950 su obra Basic Principales Of Currículo And Instruction, en la cual propone la evaluación basada en objetivos. El enfoque, sigue siendo cuantitativo, sin embargo, se amplía el espectro de los tópicos que deben ser evaluados y se perfila la necesidad de tener en cuenta las intenciones e intereses de los propios generadores y protagonistas de los programas. 
Con la época de los sesenta en la cual se presenta alguna prosperidad, se privilegia la asignación de recursos a programas exitosos, Cronbach en 1963 incorpora en la evaluación la preocupación por la recolección y el uso de la información a la que le asigna como finalidad su utilidad para la toma de decisiones; y la distinción entre evaluación formativa y evaluación sumativa. Ya con Scriven en 1967 se evidencia la necesidad de reconocer el valor de lo realizado y los condicionantes que lo pueden haber afectado. Por otro lado, a finales de la década, las corrientes de corte cualitativo se impusieron en la concepción de las ciencias sociales con una reivindicación de las especificidades humanas en la evaluación.

En esta época de globalización, en la cual, la mayor parte de los ciudadanos del mundo con un mínimo de recursos tecnológicos pueden conocer lo que pasa en cualquier parte en materia política, social, cultural, tecnológica ${ }^{6}$. etcétera, se abren nuevas perspectivas para los cambios ideológicos de las organizaciones y de la sociedad. Con ello, el futuro de las organizaciones - empresariales o no-, se centra no tanto en la experticia manual de las personas como en la creatividad y en las máximas posibilidades intelectuales que un ser humano puede desarrollar

Lo anterior supone que las personas deben encontrar en el conocimiento y en su utilización la principal forma de vinculación al sector laboral. En este sentido se podría coincidir con Malraux en que nuestra civilización se está transformando ${ }^{7}$. Estamos viviendo en una sociedad en la que están adquiriendo primacía los conocimientos teóricos y los tácitos o implícitos sobre cualquier otra clase de conocimiento; aparecen como relevantes aquellos que requieren de un determinado modelo mental y de un proceso concreto de creación intelectual, es decir, de un proceso basado en ideas, en la abstracción y en la innovación.

Es tal el avance de la nueva concepción, que hoy se habla de una economía del conocimiento en la cual se cambian los conceptos explicativos tradicionales de la economía misma: En efecto, los llamados hechos económicos asociados a la producción, el consumo y el intercambio quedan circunscritos tanto a la creación de conocimiento como a la preferencia, a la utilidad del nuevo conocimiento y a la imperfección de su mercado. Las cantidades económicas centradas en la medida de aquellas que están sujetas al intercambio, consumo y producción se preocupan por la medición de los activos intangibles.

Davenport y Prusak definen al conocimiento como una integración dinámica de experiencias, valores, información contextualizada e ideas que proveen un marco para evaluar e incorporar nuevas experiencias e información. De acuerdo a ello se puede inferir que la información constituye tan solo un insumo para el conocimiento y que tan sólo cuando pueda ser organizada por la mente de las personas se puede convertir en conocimiento. Ahora bien, el conocimiento que tienen las personas pierde validez cuando no transforma las actitudes de las personas y no sea incorporado a las instituciones en forma de documentos, rutinas, procesos, normas y prácticas organizacionales.

\footnotetext{
${ }^{6}$ Se sabe que la tecnología tiende a desarrollarse hasta tal punto que las destrezas necesarias para trabajar con dicha tecnología ya no van a ser necesarias

${ }^{7}$ André Malraux afirmaba que una civilización se transforma cuando su elemento más doloroso -humillación en el esclavo, el trabajo en el obrero moderno- se convierte, de pronto, en un valor; cuando ya no se trata de escapar a esa humillación, sino a esperar de ella la propia salvación; cuando no se trata de escapar de ese trabajo, sino de encontrar en él la propia razón de ser. Es preciso que la fábrica, que no es aún más que una especie de iglesia de catatumbas, se convierta en lo que fue la catedral, y que los hombres vean en ella, en lugar de los dioses, la fuerza humana en lucha contra la tierra..."
} 
En este modelo las actividades de enseñanza y de aprendizaje se piensan y se diseñan en función de su contribución a la plena e integral formación del estudiante como persona y como profesional. Esto implica para el profesor la construcción de escenarios en los cuales el estudiante pueda expresar todo su potencial intelectual a través de actividades que le sean significativas y le motiven al esfuerzo que enriquece y produce la satisfacción del aprendizaje.

Se requiere entonces, una perspectiva evaluativa que supere la simple descripción de lo que realiza un profesor y permita comprender toda la potencialidad de su labor y apunte a su perfeccionamiento. Ello no es posible sin romper con viejos paradigmas y con el énfasis tecnicista y el carácter positivista que la ha acompañado.

El siguiente planteamiento de Weiss: "La pregunta evaluativa se limita a buscar las metas del programa, traducirlas a indicadores mensurables de la realización de los objetivos, reunir datos de los indicadores y compararlos con criterios de la realización de las metas", demuestra la dificultad de esta empresa. Obsérvese como se reduce el problema del conocimiento al hecho fenomenológico, los hechos se imponen sobre las ideas, la medida se convierte en el elemento central de la evaluación y la objetividad se reduce al momento de la cuantificación de los datos; quedando por tanto la comprensión del hecho evaluado y la misma posibilidad teórica de la evaluación claramente limitadas.

Rist ilustra cabalmente la dificultad cuando afirma "La adhesión a un paradigma y su oposición a otro predispone a cada uno a concebir el mundo y los acontecimientos que en él se desarrollan de modos profundamente diferentes" En la fuerza que tienen los paradigmas se puede entender porque el objeto de estudio de la evaluación es rápidamente adecuado por los diferentes enfoques a los requerimientos del método experimental.

De acuerdo a lo anterior, podría afirmarse con Díaz Barriga que el paradigma cuantitativo ha sido predominante en los diferentes enfoques y que su influencia no ha perdido fuerza en la actualidad. Desde este paradigma se enfatiza la explicación en la contrastación empírica y la medición objetiva; lo objetivo está presente en el contexto de la justificación; se orienta hacia la comprobación con base en datos sólidos y repetibles sobre los cuales el evaluador debe tomar distancia para no "sesgarse" en su análisis; en su capacidad, soportada estadísticamente, de hacer inferencias basa su poder de generalización y presume la estabilidad de la realidad que se está abordando.

Siendo la medición un elemento del paradigma cuantitativo, es importante diferenciar el proceso de medición como acto comparativo de la componente métrica del aspecto. En lo métrico hay un componente técnico del cual se derivan los instrumentos y procesos de medición intrínsecos y solidarios empírica y científicamente; es decir un instrumento puede medir una propiedad porque es la propiedad misma y si no, no puede. Así la cinta métrica puede medir una longitud porqué es longitud.

Para el caso de los procesos dinámicos el concepto de equilibrio es el que prima, por ejemplo el termómetro mide la temperatura en su intercambio dinámico con el objeto que se está midiendo; sin embargo, la temperatura que arroja la medición no es la misma que se tenía antes de interactuar con el termómetro. Aplicado al campo evaluativo, dinámico por naturaleza, hacemos hincapié en que la aplicación de cualquier instrumento de medición implica desde el momento mismo de su implementación la modificación de los aspectos que se pretenden analizar, es decir, si persiste la ilusión de "medir" en el sentido métrico del término deberíamos usar instrumentos que evolucionen conforme lo hacen los aspectos 
evaluados. Dicho de otra manera, la pretensión de evaluar desde la perspectiva de la medición reafirma que la evaluación no es "inofensiva", el que pregunta de alguna manera orienta la respuesta y el que evalúa señala el deber ser del objeto evaluado.

Desde una perspectiva diferente al esquema cuantitativo y con base en los trabajos realizados por Weiss, Rein, Parlett, Hamilton, Guba surge el paradigma cualitativo centrado en la persona y en el descubrimiento del significado, en los motivos y las intenciones que ella tiene; es decir, su intención es comprensiva antes que determinística; se orienta hacia el proceso enmarcado en unas especificidades a partir de cuya singularidad no es posible establecer generalizaciones; el trabajo sobre los datos se hace desde la interacción con ellos; no basa su validez ni su fiabilidad en la "objetividad" sino en la riqueza y profundidad de sus descubrimientos.

$\mathrm{Si}$ bien se buscaba con el paradigma cualitativo romper el paradigma cuantitativo, pronto los diferentes profesionales ${ }^{8}$ de la evaluación reconocen la importancia de encontrar vías para acceder a la realidad social y empiezan a tender puentes en la búsqueda de la complementariedad y de la articulación entre ellos. Hemos llegado de esta manera a un debilitamiento de la frontera entre estos dos paradigmas que ha permitido la indagación investigativa en torno a la evaluación, pues como sostiene House "En los últimos años ha surgido como un campo de actividad por derecho propio, con un amplio conjunto de modelos $y$ enfoques $y$ con sus propios criterios, principios y normas".

Lo cierto es que, en la práctica los paradigmas son eclécticos pues se utilizan diversas técnicas y métodos en la recolección, análisis e interpretación de la información independientemente del tipo de paradigma al cual se encuentran vinculados. La tendencia actual en la evaluación educativa se orienta por un énfasis cualitativo, hay consenso en que si no es cualitativa no es educativa, pero con fuerte apoyo en las técnicas y procedimientos de tipo cuantitativo. Se reconoce la necesidad de dar una mayor solidez a la recolección y una mayor posibilidad de interpretación a la información sin perder de vista el carácter formativo de la evaluación y la dimensión social que esta tiene.

En suma no hay ruptura radical ni de desplazamiento y reemplazo de un paradigma por el otro, lo actual es más bien la complementariedad de ambos.

\section{LA DIMENSIÓN SOCIAL DE LA EVALUACIÓN}

Queda claro entonces que en la evaluación debe tenerse en cuenta, además de las particularidades del proceso, los marcos políticos, sociales y culturales en los cuales se llevará a cabo y, en lo posible, cuidar de las consecuencias que el ejercicio tendrá no solo para las personas sino para la sociedad en general. Edgar Morin en sus trabajos sobre pensamiento complejo advierte que en este mundo tan globalizado, cualquier acto humano por inocente que parezca puede tener repercusiones importantes en cualquier escenario mundial. $\mathrm{Y}$ a esta idea le podemos agregar un matiz de tipo dialéctico con lo que sostiene Díaz Barriga "De alguna manera se puede hablar de que la evaluación es condicionada socialmente, a la vez que por sus resultados condiciona a la sociedad".

\footnotetext{
${ }^{8}$ Stufflebeam afirma que a partir de 1973, por diferentes motivos comienza un período de profesionalismo de la evaluación. Citado por Franco, N. y Ochoa, L. En La racionalidad de la acción en la evaluación, Ed. Magisterio, Bogotá, Colombia, 1997, pp. 16

${ }^{9}$ Citado por Helen Simons en Evaluación Democrática de Instituciones Escolares. Morata, Madrid, 1999, pp. 26
} 
Este carácter social es el que permite ver a la evaluación como un campo de poder que rebasa la simple dimensión técnica y que está pletórico de intencionalidades. Obliga a pensar, por ejemplo, cómo desde la evaluación de las instituciones educativas se intenta legitimar políticas y a justificar las limitaciones del Estado para cumplir con su compromiso de país.

Es fácil, desde esta perspectiva entender cómo, desde prácticas evaluativas orientadas hacia el control, un gobierno podría justificar la negación de recursos a las universidades públicas o deteriorar las condiciones laborales de los docentes merced a "los malos resultados obtenidos".

Por ejemplo, en Colombia, una evaluación de corte diagnóstico de tipo cuantitativo evidenció problemas de cobertura, menor cantidad de horas escolares que en otros países y una inferior ratio profesor alumno. Con base en ello, el gobierno trazó la política de fusión ${ }^{10}$ de instituciones escolares, aumento de la jornada laboral para los maestros y reubicación de profesores y estudiantes, sin analizar detenidamente las implicaciones pedagógicas del hecho o los aumentos en costos de transporte de las personas reubicadas, problemas de seguridad incrementados con los traslados, ni las dificultades de gobernabilidad de esas instituciones; en otras palabras, sin importar el efecto sobre la calidad educativa de los estudiantes ni sus condiciones reales de permanencia en el sistema escolar.

Saber para qué se evalúa, a quién le interesa y a quién sirve la evaluación ayudará a la comprensión del fenómeno evaluativo y permitirá entender que no es posible explicar los resultados obtenidos atendiendo sólo a las características intrínsecas del objeto de evaluación. Una evaluación construida e interpretada sólo desde el objeto de la evaluación además de ser totalmente errónea, constituye una aberración de lamentables consecuencias para el mejoramiento educativo y por ende para el desarrollo de nuestros pueblos.

\section{EL PROBLEMA DE LA VALIDEZ Y LA FIABILIDAD}

En términos generales existe una estrecha relación entre los conceptos de validez y confiabilidad ya que ambos buscan determinar los grados y niveles de representatividad de la realidad, ya sea desde una dimensión objetiva o subjetiva ${ }^{11}$. Referidas y asociadas a la credibilidad de un determinado proceso, todo evaluador se siente en la obligación de argumentar acerca de la validez y la fiabilidad de su ejercicio; no pocos evaluadores recurren a la utilización de alguno de los tipos de validez existentes en la literatura para demostrar como se cumple con algún criterio asociado a la misma.

En principio, los criterios desde los cuales se examina la validez o la fiabilidad de una propuesta están determinados por él o por los paradigmas bajo los cuales se cobija. Con respecto a los paradigmas cuantitativo y cualitativo, algunos expertos afirman que ninguno garantiza la validez, pues ella se enmarca en la discusión entre lo que es objetivo y lo que es subjetivo. A los procedimientos cuantitativos se les critica que los criterios son externos al proceso mismo y hablan más de la posibilidad de replica del modelo que a su credibilidad, mientras que, a los cualitativos se les acusa de una falta de rigurosidad derivada de la reubicación en ocasiones demasiado flexible de las creencias de esos mismos participantes.

\footnotetext{
${ }^{10}$ Se unen varias instituciones para tener grupos más numerosos de alumnos, con ello se liberan plazas de docentes y eliminan puestos directivos.

${ }^{11}$ Algunos autores asocian el concepto de validez al paradigma cualitativo y el de confiabilidad al cuantitativo
} 
Karl Popper ubica el problema de la objetividad en la búsqueda de hechos que desmientan o contradigan la teoría, Horkheimer y Adorno de la Escuela de Frankfurt, señalan que la sociedad mo puede considerarse como un objeto más, la ciencia también es subjetiva y por tanto la objetividad se alcanza con el método crítico, esta escuela afirma categóricamente que lo objetivo es el acuerdo o consenso entre sujetos que han de ser críticos y entendidos en un campo determinado de la ciencia y del conocimiento real. Con diferencias Durheim, Goldman y Weber ubican el problema de la objetividad en la mediación de los juicios de valor.

Una de las principales características para la validez en las ciencias humanas es su capacidad para demostrar que las proposiciones generadas se ajustan a las condiciones reales que rigen en la vida institucional. La utilización de diferentes fuentes así como la exploración de diversas vías para obtener información como mecanismo de triangulación contrastada son estrategias fundamentales para garantizar confiabilidad y validez en el ejercicio. Resulta valioso y muy iluminador para la evaluación el planteamiento de Cardona: "El conocimiento científico exige como vía de elaboración la subjetividad crítica y racional y no el subjetivismo parcial y tendencioso".

Denzin en 1970, Silverman en 1985, Fielding en 1986, niegan que el conocimiento obtenido por triangulación sea necesariamente más objetivo y fiable que el obtenido mediante una única metodología. En la misma dirección Fernández Huerta afirma "No es posible construir objetividades a partir de la interrelación de intersubjetividades". No obstante es un hecho que la triangulación favorece la adquisición de un conocimiento más amplio y profundo de la realidad social, con lo cual se impide la aceptación fácil de los datos e impresiones iniciales, se amplía el ámbito, la densidad y la claridad de los conceptos y se ayuda a corregir los sesgos.

\section{EVALUACIÓN DEL DOCENTE UNIVERSITARIO}

Atendiendo a la concepción impulsada por Vain en el sentido de que la docencia universitaria se ejerce desde una compleja red en la que coexisten múltiples entrecruzamientos y diversas tensiones, se asume la evaluación del docente universitario desde una perspectiva institucional en el entendido de que a partir de sus definiciones se establece el escenario desde el cual todo profesor puede construir su identidad profesional y su proyecto de vida académica.

Las instituciones universitarias alrededor de su misión edifican un proyecto institucional en el cual patentizan sus principios, propósitos y estrategias que abarcan campos tan significativos como el de la docencia, la investigación y la proyección social. Las estructuras organizativas, los niveles jerárquicos, las rutinas comunicativas, los esquemas de participación, establecidos como un todo articulado constituyen el ambiente institucional desde el cual se posibilita el quehacer docente.

Anteriormente se ha insistido en la dimensión social de la evaluación y en la necesidad de establecer el para qué y a quién sirve la evaluación. En este sentido es necesario dejar sentado que todo acto evaluativo debe estar pensado desde una perspectiva de mejoramiento y construido para beneficiar a la sociedad como un todo.

Por ello, el proceso evaluativo debe privilegiar una profunda reflexión en torno a las posibilidades de mejoramiento como arma esencial para potenciar la capacidad transformadora que coadyuve a la solución de las más sentidas problemáticas siempre en pro de una sociedad más justa y humana. Como sostiene 
Medina Rivilla " La evaluación es la actividad reflexiva que nos permite conocer la calidad de los procesos y los logros alcanzados en el desarrollo del proyecto. La evaluación es una valoración sistemática que facilita el conocimiento minucioso de los procesos aplicados y fundamentalmente las decisiones futuras de cambio que nos proponemos llevar a cabo".

Un análisis de nuestras instituciones de educación superior muestra una significativa clasificación de los profesores en docentes ${ }^{12}$, investigadores y profesores que cumplen funciones de proyección social. Con ello se inserta además de una clara división del trabajo, una jerarquización injustificada de la profesión docente.

Como consecuencia de lo anterior, los generadores de conocimiento aparecen alejados de quienes deben transmitirlos y por supuesto de quienes por algún designio institucional emergen como los encargados de vincularlos a los desarrollos de sectores específicos de la sociedad. Esta separación conlleva a su vez a clasificar a las Instituciones como instituciones de docencia o de investigación, según el énfasis que se les asigne.

En una investigación realizada en $1999^{13}$ había demostrado que las instituciones de educación superior en Colombia ${ }^{14}$ centraban la evaluación profesoral en las informaciones obtenidas de la aplicación rutinaria de encuestas de opinión a los alumnos. Esta práctica, que aún subsiste ejemplifica como se privilegia institucionalmente la docencia sobre las demás funciones sustantivas de las Universidades, no debe olvidarse que el énfasis evaluativo señala con claridad las prácticas culturales y organizativas que se fomentan.

Este modelo evaluativo ha hecho crisis en nuestras instituciones y como resultado la evaluación del profesor universitario, aparece con unos niveles de descrédito que impiden utilizar todo su potencial transformador. La desacreditación emerge principalmente desde las dos fuentes comprometidas:

Por la vía de los estudiantes: ellos consideran que se debe evaluar para mejorar la calidad de sus profesores y no entienden como, a pesar de calificar mal a un profesor este vuelve a dictarles clase en los próximos semestres y peor aún con las mismas fallas que ellos habían evidenciado. Para los estudiantes es imperativa la ecuación: "profesor mal evaluado, profesor retirado". Si ello no se da la evaluación no es útil y por tanto los cuestionarios no sirven para nada y terminan por contestarlos sin mayor responsabilidad.

Por la vía profesoral: no se entiende como puede ser posible que la institución los ponga 'contra al paredón' frente a unos estudiantes cuyos elementos de análisis son por lo menos cuestionables y que la mayoría de las veces responden los cuestionarios acorde con la forma como les esté yendo en las calificaciones del curso. La estrategia de "hagámonos pasito" en la cual el profesor no evalúa mal a los estudiantes para que estos no lo evalúen mal es el mayor peligro que se cierne sobre la educación en este modelo evaluativo.

Por supuesto que no se trata de abandonar a los alumnos como fuente valida de información para la evaluación de los profesores; hacerlo sería tanto como abandonar la responsabilidad que tiene la institución de garantizar procesos de enseñanza - aprendizaje pertinentes y acordes con las necesidades de formación de nuestros ciudadanos. Lo que se pretende es reconocer que no es desde la crítica

\footnotetext{
${ }^{12}$ La palabra docente se asocia comúnmente con el papel de enseñanza que cumple el profesor

${ }^{13}$ Estudio realizado con 54 instituciones universitarias de Colombia

${ }^{14}$ No sería difícil demostrar que pasa igual en la gran mayoría de los países latinoaméricanos
} 
contemplativa y perceptual de la labor ni desde la inercia pedagógica, entendida como el continuar con las mismas prácticas, que se logra alcanzar una cualificación de la enseñanza y el aprendizaje.

Dos elementos consideramos esenciales para articular una propuesta de evaluación profesoral: a) entender que el desempeño profesoral debe asumirse en un contexto que supera las características intrínsecas del profesor y b) fortalecer los procesos de triangulación.

El primero lejos de desconocer que la docencia se mejora desde el trabajo del profesor, invita a entender las dinámicas institucionales que generan las condiciones necesarias para ejercer una docencia de calidad y determina la implementación de estrategias que posibilitan su cualificación.

El segundo aboga por la utilización de diferentes fuentes, perspectivas y vías de indagación y análisis que permita una mirada más integral al trabajo profesoral. Por la vía de las fuentes, es necesario caracterizarlas en función de la pertinencia que tenga para el proceso, las perspectivas se relacionan con los enfoques que van a orientar el proceso y las vías de indagación aluden a los mecanismos de obtención, organización y análisis de la información que servirá de base para la identificación de fortalezas y posibilidades de mejoramiento.

Por ejemplo, al analizar a los estudiantes como fuente de información pueden surgir interrogantes acerca de qué información pueden proporcionar, en qué momentos serán consultados como se valorará la información obtenida etc. se deben resolver cuestiones como las siguientes: ¿ A todos los estudiantes se les puede interrogar sobre todos los aspectos del trabajo de sus profesores? ¿Valdría la pena presentar cuestionarios de opinión diferentes para estudiantes de primeros semestres que para los de semestres intermedios o para los de últimos semestres? ¿ Los estudiantes sólo pueden dar información válida sobre los procesos de enseñanza - aprendizaje? ¿Es necesario cruzar información de rendimiento de los estudiantes con la información obtenida de sus percepciones acerca del trabajo del profesor? ¿El rendimiento de los estudiantes es un buen indicador del trabajo del profesor? ¿Si a los estudiantes les va bien es mérito del profesor y si les va mal se debe a un mal trabajo del profesor? Etc.

Con respecto a los colegas deberían resolverse entre otras cuestiones como las siguientes: ¿Qué condiciones deben generarse para lograr que los profesores suministren información confiable sin sentir que faltan a su ética profesional? ¿Sobre qué aspectos pueden ser interrogados los profesores acerca del trabajo de sus colegas? ¿Todos los profesores pueden suministrar información sobre los mismos aspectos? ¿Qué impacto tiene en el ambiente organizacional la evaluación realizada por los colegas? ¿Son convenientes los cuestionarios en los que se pide una calificación a los profesores?. Y así sucesivamente se procede a caracterizar cada una de las fuentes que se vayan a utilizar.

En cuanto al proceso en su totalidad es importante clarificar interrogantes tales como: ¿Quiénes participan y como lo hacen en la valoración integral del trabajo profesoral? ¿Es conveniente generar una especie de escalafón de los mejores profesores? ¿Es necesario ponderar los diversos frentes del desempeño profesoral? ¿Cómo lograr el sano equilibrio entre la autocomplacencia y la autoflagelación? Y muchos otros más. 


\section{HACIA LA CONSOLIDACIÓN DE UN MODELO DE EVALUACIÓN PROFESORAL ${ }^{15}$}

La sociedad actual exige que las universidades conjuguen adecuadamente sus tres funciones sustantivas y por ello se requiere con mayor urgencia que el profesor universitario, investigue enseñe y se proyecte socialmente. Para ello, se requiere fundamentalmente de dos cosas: Del desarrollo profesional de los docentes ligado a su contexto particular y de la mejora de las prácticas educativas.

El primero va necesariamente ligado a sus procesos de capacitación y actualización docente tanto en el plano pedagógico como en el disciplinar, a la reflexión que haga de su propia labor docente y a su participación decidida en la reflexión institucional que adquiere necesario vigor en el ejercicio autoevaluativo. El segundo alude directamente al currículo que, tal como lo plantean Medina y Blanco es un campo de investigación en la que se conjugan teoría y práctica y una acción incitadora y explícita de la profesionalización de los docentes y la formación de los discentes. Desde el currículum se da significado al qué, cómo y para qué y el porqué del proceso de enseñanza que promueve aprendizaje

La evaluación profesoral desde la perspectiva institucional abarca como campos de acción para el trabajo de los profesores los de la docencia, la investigación, la proyección social, la gestión y el desarrollo profesoral, concebidos de la siguiente manera:

- La docencia esta conformada por las actividades necesarias para impartir un grupo de una asignatura de pregrado o postgrado, en los períodos semestrales normales o en a período intermedio, las cuales incluyen: la clase presencial, la preparación de clase, la evaluación de los estudiantes y su correspondiente realimentación con fines de orientación y la atención fuera del aula a los estudiantes del grupo. Así mismo se considera actividad de docencia la tutoría o consejería a un grupo de estudiantes.

- La investigación está conformada por las actividades necesarias para generar conocimiento en cualquiera de sus posibilidades, las cuales incluyen: la planeación y ejecución de proyectos específicos y la presentación, confrontación y difusión de los resultados del proyecto. Así mismo se considera actividad de investigación la dirección y/o asesoría de proyectos de grado, el apoyo, mediante asesoría especializada, a proyectos formales de investigación y la producción intelectual.

- La proyección social está conformada por actividades relacionadas con proyectos para aplicación de conocimientos, educación continuada, proyectos de beneficio social y el manejo de relaciones con comunidades académicas nacionales e internacionales.

- La gestión académica está conformada por actividades de planeación, decisión, ejecución, control y orientación de Facultades o Departamentos académicos, o de Dirección de Programas académicos de pregrado o postgrado o de Coordinación académica de áreas del conocimiento desarrolladas por el Departamento o de Dirección de Centros de soporte académico que hayan sido establecidos como tales.

\footnotetext{
15 Este modelo se desarrolla en la Universidad Autónoma de Occidente de Cali Colombia. Presentado como propuesta de consolidación debe entenderse que algunas de sus componentes todavía no han sido incorporadas en su totalidad.
} 
- El desarrollo profesoral está conformado por actividades de capacitación y desarrollo del profesor, tendientes a mejorar su actividad académica.

Con base en estas definiciones, se estructura el modelo de evaluación profesoral que desarrolla los siguientes elementos:

\section{Identificación de los elementos referenciales:}

1.1. La institución define un Plan de Desarrollo a partir del cual se establecen los planes de desarrollo de las Facultades y los Departamentos. Estos planes de desarrollo generan los insumos fundamentales que permiten construir las agendas de trabajo semestral.

1.2. Las agendas semestrales de las Facultades y los Departamentos y los interesés y necesidades de desarrollo de cada profesor, sirven de marco a partir del cual, cada profesor acuerda un plan de trabajo semestral con su jefe inmediato.

1.3. Estos planes de trabajo incluyen activi dades de docencia, investigación, proyección social, gestión y desarrollo profesoral, constituyen el principal referente evaluativo para el profesor. Es decir, al profesor se le evalúa principalmente en función de lo que se compromete a realizar, y a su vez desde los planes semestrales de trabajo, se identifican las potencialidades del docente y el grado de articulación existente entre las diferentes instancias institucionales.

\section{Identificación de las fuentes e instrumentos:}

Para la evaluación del desempeño docente se utilizan las siguientes fuentes:

2.1. Los alumnos: Con ellos se obtiene información especialmente desde dos vías:

2.1.1. Se recogen opiniones acerca de la forma como el docente desarrolla sus procesos de enseñanza y de aprendizaje. Se utilizan, para ello, dos tipos de procedimientos:

2.1.1.1. Coevaluación: Mediante la cual, estudiantes y profesor, establecen un diálogo acerca de los aspectos positivos y los negativos que han aparecido en el curso y construyen una propuesta de mejoramiento que plasman en un acta de compromiso. Este instrumento de corte cualitativo se aplica entre la semana 4 y la 6 del semestre académico.

Las respectivas actas se organizan en cada unidad académica y son los coordinadores de área los encargados de hacer seguimiento al curso. Al final del semestre, se utilizan como insumo para interpretar los resultados obtenidos en el cuestionario de opinión y construir diálogos, entre los docentes del área, en torno a la manera de realizar una mejor docencia.

2.1.1.2. Cuestionario de opinión: Conjunto de preguntas, que indagan sobre distintos aspectos del trabajo docente. Los estudiantes de primeros semestres plasman sus opiniones en relación con cumplimiento, metodología y evaluación del aprendizaje; los estudiantes de últimos semestres opinan además sobre dominio de asignatura y pertinencia de las temáticas tratadas. Con la información obtenida se realizan las siguientes acciones: 
2.1.1.2.1. Procesada la información se elabora un informe a los departamentos en el cual se señalan los resultados obtenidos por todos sus docentes.

2.1.1.2.2. Se envía a cada profesor un informe sobre sus resultados, se explica el sentido de la evaluación y se le hacen sugerencias de mejoramiento.

2.1.1.2.3. Se entrega un informe a cada docente de las sugerencias y comentarios que consignan los estudiantes para mejorar su desempeño.

2.1.1.2.4. Semestralmente, se entrega a cada departamento un informe histórico de los resultados obtenidos por cada profesor durante los semestres en los cuales ha dictado clase en la institución. Con este informe, se identifica a los docentes que son consistentes en sus resultados tanto positivos como negativos, para reforzar y estimular los buenos desempeños y establecer estrategias de mejoramiento con los de deficiente desempeño.

2.1.1.2.5. Análisis estadísticos para establecer correlaciones, modelos de regresión etc. con el propósito de retroalimentar a las unidades académicas acerca de los aspectos que, en opinión de los alumnos, más influyen en el desempeño de sus docentes. En este sentido y después de realizar diferentes análisis en diferentes semestres se han consolidado las siguientes conclusiones:

- El hecho de que un profesor sea cumplido en sus horarios, no parece afectar la valoración que de él hacen sus estudiantes en otras variables. En cambio si el profesor es incumplido resulta mal evaluado en las preguntas relacionadas con metodología.

- El elemento que más afecta la calificación que asignan los estudiantes al desempeño profesoral es el de su claridad expositiva.

- Las aplicaciones que un docente realiza de su disciplina en otros campos del saber, impacta positivamente la participación de sus estudiantes en las clases.

- La variable cumplimiento tiene una mayor importancia para los estudiantes de ingeniería que para los estudiantes de carreras como comunicación social.

- Para estudiantes de comunicación social, publicidad y mercadeo la variable relaciones con los estudiantes tiene una gran importancia en el momento de valorar el trabajo profesoral

- Para los estudiantes de Ingeniería y ciencias económicas y administrativas es muy importante la variable dominio que demuestre el profesor sobre su asignatura.

- Los factores que más influyen en el desempeño profesoral desde la perspectiva de los estudiantes son: la claridad expositiva, la claridad con la que responde a los interrogantes planteados por los estudiantes, la justicia con la cual asigna sus calificaciones.

- En general los factores que menos influyen en el desempeño profesoral desde la perspectiva de los estudiantes son: la coherencia entre la bibliografía recomendada y el desarrollo del curso, la asistencia puntual a las clases, si los contenidos se han desarrollado según los tiempos establecidos y si el profesor es atento y facilita la comunicación con los estudiantes. 
2.1.2. Exámenes de tipo comprehensivo por asignatura: Son pruebas de final de curso realizadas al termino del semestre. Además de la importancia que tienen estas pruebas en los procesos de enseñanza-aprendizaje, desde la perspectiva de la evaluación profesoral se utilizan para la reestructuración metodológica de los cursos y mediante el criterio de norma la identificación de las temáticas con bajos niveles de apropiación en los cursos dictados por el profesor.

Con esta información tanto el grupo de profesores del área como cada profesor en forma individual reflexionan acerca de los aspectos que deben ser mejorados tanto desde el punto de vista didáctico como desde los aspectos cognitivos que deben ser tenidos en cuenta en forma previa antes del desarrollo de las temáticas a tratar.

2.2. El jefe inmediato: Opinión sobre el desempeño general del docente. Se utilizan dos tipos de instrumentos:

2.2.1. Cuestionario de Opinión acerca de algunos ítem agrupados en tres campos: cumplimiento, desempeño académico, relaciones universitarias.

2.2.2. Formato de actividades en el cual se señalan todas las actividades que figuran en el plan semestral de trabajo del docente. El jefe inmediato informa del cumplimiento de dichas actividades y realiza o puede solicitar a instancias específicas que se realice alguna evaluación acerca de la calidad de las mismas.

Los aspectos relacionados con los proyectos de investigación, y producción intelectual son evaluados a través de la Vicerrectoría de Investigaciones por pares tanto internos como externos.

2.3. Colegas: Los profesores contribuyen con la identificación de fortalezas y aspectos en los cuales sus colegas deben mejorar, relacionados con cumplimiento, desempeño académico y relaciones institucionales. Si el profesor trabaja en proyectos específicos con otros docentes se pide la opinión de ellos con respecto a la participación del profesor en los mismos.

2.4. Autoevaluación: Como el principal referente evaluativo es el plan semestral de trabajo, se pide a cada profesor que informe acerca del nivel de cumplimiento de cada actividad acordada. Esta información, es contrastada con la suministrada por el Jefe inmediato y en caso de no existir coincidencia, se toman como referentes los indicadores de logro previamente establecidos para hacer las clarificaciones del caso.

\section{Tratamiento de la información:}

Se establecen las siguientes estrategias:

3.1. Toda la información es recolectada y organizada desde el Centro de Desarrollo Académico quién presenta un documento con una visión integrada de dicha información. Este documento es analizado en forma conjunta entre el profesor, el jefe de área y el jefe de departamento en un diálogo constructivo que concluye con un plan de acción conjunto tendiente a reafirmar las fortalezas y superar las dificultades detectadas.

Este plan de acción incluye los apoyos que institucionalmente recibirá el docente. 
3.2. El consejo académico de Facultad en el cual participan profesores, estudiantes y egresados, analiza un informe general organizado por el Centro de Desarrollo Académico. Este informe recoge características generales que permiten reflexionar acerca de la calidad del trabajo docente y las necesidades de desarrollo. Con base en ello se diseñan los programas de capacitación profesoral tanto a nivel disciplinario como pedagógico.

\section{Estudios de impacto:}

Anualmente, el modelo de evaluación profesoral es evaluado desde los indicadores de impacto, los cuales presentan información acerca de los mejoramientos que se han obtenido de su implementación. El consejo académico de la institución analiza y decide sobre aquellos aspectos que dificultan la implementación del modelo y que obstaculicen la feliz realización de los planes de acción establecidos.

\section{BIBLIOGRAFÍA}

ANDÚJAR José, Metodología Innovadora de Centros Educativos. Madrid, Sanz y Torres, (1994):

CASANOva María Antonia. Manual de evaluación educativa, México, Ed. La Muralla (1995)

DAVENPORT , T; Prusak, L: Working knowledge: how organizations manage what they knowBoston, Harvard business school press,. (1998

DíAZ, Mario. Didáctica y Currículo, Bogotá, magisterio,1998

FERNÁNDEZ, José. Tácticas rápidas de triangulación estimativa cualitativa y cuantitativa, guía curso de doctorado

Medina RiviLLA, Antonio (1991): Bases para un Diseño Curricular de Centro y Aula, Centro Asociado de Córdoba. UNED

RIST, R.C. On the relation among educational research paradigm: from disdain to détente, en Anthropology and education quarterly, No. 8

Rızo Héctor: La evaluación del docente univesitario. Revista Electrónica Universitaria de formación del profesorado 1999

VAIN, Pablo. La evaluación de la docencia universitaria: un problema complejo, Buenos Aires,2004

WEISS, C. Investigación evaluativa, México, Ed. Trillas 1975 


\title{
Contactar
}

Revista lberoamericana de Educación

\author{
Principal OEI
}

